\title{
Bayesian Inference for Hybrid Discrete-Continuous Stochastic Kinetic Models
}

\author{
Chris Sherlock ${ }^{1}$, Andrew Golightly ${ }^{2 *}$ and Colin S. Gillespie ${ }^{2}$ \\ ${ }^{1}$ Department of Mathematics and Statistics, Lancaster University, UK \\ ${ }^{2}$ School of Mathematics \& Statistics, Newcastle University, UK
}

\begin{abstract}
We consider the problem of efficiently performing simulation and inference for stochastic kinetic models. Whilst it is possible to work directly with the resulting Markov jump process, computational cost can be prohibitive for networks of realistic size and complexity. In this paper, we consider an inference scheme based on a novel hybrid simulator that classifies reactions as either "fast" or "slow" with fast reactions evolving as a continuous Markov process whilst the remaining slow reaction occurrences are modelled through a Markov jump process with time dependent hazards. A linear noise approximation (LNA) of fast reaction dynamics is employed and slow reaction events are captured by exploiting the ability to solve the stochastic differential equation driving the LNA. This simulation procedure is used as a proposal mechanism inside a particle MCMC scheme, thus allowing Bayesian inference for the model parameters. We apply the scheme to a simple application and compare the output with an existing hybrid approach and also a scheme for performing inference for the underlying discrete stochastic model.
\end{abstract}

Keywords: Stochastic kinetic model, linear noise approximation, Poisson thinning, particle MCMC

\section{Introduction}

A growing realisation of the importance of stochasticity in cell and molecular processes (McAdams \& Arkin 1999, Kitano et al. 2001, Swain, Elowitz \& Siggia 2002, for example) has stimulated the need for efficient methods of inferring rate constants in stochastic kinetic models (SKMs) associated with gene regulatory networks. Such inferences are typically required to allow predictive in silico experiments. Performing inference for the Markov

\footnotetext{
*andrew.golightly@ncl.ac.uk
} 
jump process representation of the SKM is straightforward given observations on all reaction times and types. In this case, it is possible to construct a complete data likelihood, for which a conjugate analysis is possible (Wilkinson 2012). In practice, a subset of species may be observed at discrete times. Boys, Wilkinson \& Kirkwood (2008) show that it is possible to construct Metropolis-Hastings schemes for performing inference in this setting. However, the statistical efficiency of such schemes can be poor, and these methods are likely to be more computationally demanding than simulating the process exactly (using, for example, the Gillespie algorithm (Gillespie 1977)). Therefore, whilst inference in this setting is possible in theory, in practice computational cost precludes analysis of systems of realistic size.

Considerable speed-up can be obtained by ignoring discreteness and stochasticity in the inferential model. For example, the macroscopic rate equation (MRE) models the dynamics with a set of coupled ordinary differential equations (van Kampen 2001). Computational savings can still be made when adopting the diffusion approximation or chemical Langevin equation (CLE) (Gillespie 2000) on the other hand, which ignores discreteness but not stochasticity by modelling the biochemical network with a set of coupled stochastic differential equations (SDEs). Although the transition density characterising the process under the CLE is typically intractable, it has been shown that basing inference algorithms around this model can work well for some applications (Golightly \& Wilkinson 2005, Heron, Finkenstadt \& Rand 2007, Purutcuoglu \& Wit 2007, Golightly \& Wilkinson 2011, Picchini 2013). Further computational gains can be made by adopting a linear noise approximation (LNA) of the CLE (van Kampen 2001, for example) which is given by the MRE plus a stochastic term accounting for random fluctuations about the MRE. Under the LNA, the transition density is a tractable Gaussian density (provided that the initial value is fixed or follows a Gaussian distribution). Performing inference for the LNA has been the focus of Komorowski, Finkenstadt, Harper \& Rand (2009), Stathopoulos \& Girolami (2013) and Fearnhead, Sherlock \& Giagos (2014) among others. However, biochemical reactions describing processes such as gene regulation can involve very low concentrations of reactants (Guptasarma 1995) and ignoring the inherent discreteness in low copy number data traces is clearly unsatisfactory.

The aim of this paper is to exploit the computational efficiency of methods such as the CLE and LNA whilst accurately describing the dynamics of low copy number species. Hybrid strategies for simulating from discrete-continuous stochastic kinetic models are reasonably well developed and involve partitioning reactions as fast or slow based on the likely number of occurrences of each reaction over a given time interval and the effect of each reaction on the number of reactants and products. Use of the CLE to model fast reaction dynamics in order to simulate efficiently from an approximation to the system has been the focus of Haseltine \& Rawlings (2002), Burrage, Tian \& Burrage (2004), Salis \& Kaznessis (2005) and Higham, Intep, Mao \& Szpruch (2011) amongst others. Discrete/ODE approaches (e.g. Kiehl, Matteyses \& Simmons (2004) and Alfonsi, Cances, Turinici, Ventura \& Huisinga (2005)) are also possible and we refer the reader to Pahle (2009) and Golightly \& Gillespie (2013) for recent reviews. Since the slow reaction hazards will necessarily depend on species involved in fast reactions, these hazards are typically not constant between slow reaction events, and efficient sampling of these slow event times can be problematic.

We propose a novel hybrid simulation strategy that models fast reaction dynamics with the LNA and slow dynamics with a Markov jump process. Moreover, by deriving a probable upper bound for a combination of components that drive the LNA, we obtain a probable 
upper bound for the total slow reaction hazard. This allows efficient sampling of the slow reaction times via thinning, which is a point process variant of rejection sampling (Lewis \& Shedler 1979). Related approaches have been proposed by Casella \& Roberts (2011) and Rao \& Teh (2013). The former consider simulation for jump-diffusion processes by combining a thinning algorithm with a generalisation of the exact algorithm (for diffusions) developed by Beskos \& Roberts (2005), whilst the latter assume that an upper bound for the rate matrix governing the MJP is available and use uniformisation (Hobolth \& Stone 2009) to simulate the process.

We use our approximate model to perform Bayesian inference for the governing kinetic rate constants using noisy data observed at discrete time points. In particular, we focus on a special case of the particle marginal Metropolis Hastings (PMMH) algorithm (Andrieu, Doucet \& Holenstein 2010) which targets the marginal posterior density of the model parameters and permits exact, simulation-based inference. The algorithm requires implementation of a particle filter (Carpenter, Clifford \& Fearnhead 1999, Pitt \& Shephard 1999, Doucet, Godsill \& Andrieu 2000, Del Moral, Jacod \& Protter 2002) in the latter step, and we apply the bootstrap filter (Gordon, Salmond \& Smith 1993) which only requires the ability to forward simulate from the model and evaluate the observation densities associated with each data point. Use of our novel hybrid simulator inside the filter therefore avoids the need to evaluate the transition density associated with the hybrid model. We believe that this is the first serious attempt to explore the performance of a hybrid simulator when used as an inferential tool.

To validate the methodology, we apply the method to an autoregulatory process with five reactions and two species. This simple application allows comparison of the proposed hybrid inference scheme with a scheme for performing inference for the true underlying discrete stochastic model. Finally, we compare the performance of the proposed hybrid scheme as an inferential tool with an approach based upon the simulation methodology described in Salis \& Kaznessis (2005).

The remainder of the article is structured as follows. In Section 2 we give a brief exposition of the stochastic approach to chemical kinetics before outlining the hybrid simulation technique in Section 3. Section 4 describes the particle MCMC scheme for inference. This is then applied in Section 5 before conclusions are drawn in Section 6 .

\section{Stochastic Kinetics - A Brief Review}

We consider here the stochastic approach to chemical kinetics and outline a Markov jump process (MJP) description of the dynamics of a system of interest, expressed by a reaction network. Two approximations that can be used in a hybrid modelling approach are outlined. For further details regarding stochastic kinetics we refer the reader to Wilkinson (2012).

\subsection{Stochastic Kinetic Models}

A biochemical network is represented with a set of reactions. We have $k$ species $\mathcal{X}_{1}, \mathcal{X}_{2}, \ldots, \mathcal{X}_{k}$ and $r$ reactions $R_{1}, R_{2}, \ldots, R_{r}$ with a typical reaction $R_{i}$ of the form,

$$
R_{i}: \quad u_{i 1} \mathcal{X}_{1}+\ldots+u_{i k} \mathcal{X}_{k} \stackrel{c_{i}}{\longrightarrow} v_{i 1} \mathcal{X}_{1}+\ldots+v_{i k} \mathcal{X}_{k}
$$


Note that $c_{i}$ is the kinetic rate constant associated with reaction $R_{i}$ and we write the vector of all rate constants as $\mathbf{c}=\left(c_{1}, c_{2}, \ldots, c_{r}\right)^{\prime}$. Clearly, the effect of reaction $i$ on species $j$ is to change the number of molecules of $\mathcal{X}_{j}$ by an amount $v_{i j}-u_{i j}$. To this end, we may define the $r \times k$ net effect matrix $\mathbf{A}$, given by $\mathbf{A}=\left\{a_{i j}\right\}$ where $a_{i j}=v_{i j}-u_{i j}$. To induce a compact notation, let $\mathbf{X}(t)=\left(X_{1}(t), X_{2}(t), \ldots, X_{k}(t)\right)^{\prime}$ denote the number of molecules of each respective species at time $t$. Now, under the assumption of mass action kinetics, the instantaneous hazard of $R_{i}$ is

$$
h_{i}\left(\mathbf{X}(t), c_{i}\right)=c_{i} \prod_{j=1}^{k}\left(\begin{array}{c}
X_{j}(t) \\
u_{i j}
\end{array}\right)
$$

The order of reaction $i$ is $\sum_{j} u_{i j}$. The evolution of a biochemical network of interest is most naturally modelled as a Markov jump process. Whilst the transition density associated with the process typically does not permit analytic tractability, the process can be exactly simulated forwards in time using a discrete event simulation method. The most well-used method is known in the stochastic kinetics literature as the Gillespie algorithm (Gillespie 1977) and uses the fact that if the current time and state are $t$ and $\mathbf{X}(t)$ respectively then the time $\tau$ to the next reaction event is

$$
\tau \sim \operatorname{Exp}\{\lambda(\mathbf{X}(t), \mathbf{c})\}, \quad \text { where } \quad \lambda(\mathbf{X}(t), \mathbf{c})=\sum_{i=1}^{r} h_{i}\left(\mathbf{X}(t), c_{i}\right),
$$

and the reaction that occurs will be type $R_{i}$ with probability proportional to the reaction hazard $h_{i}\left(\mathbf{X}(t), c_{i}\right)$. Other exact simulation methods are possible - Gibson and Bruck's next reaction method (Gibson \& Bruck 2000) is widely regarded to be the most computationally efficient strategy. As these methods capture every reaction occurrence, they can be extremely computationally costly for many systems of interest.

\subsection{Chemical Langevin Equation}

The CLE (van Kampen 2001, Golightly \& Wilkinson 2005) can be constructed by calculating the infinitesimal mean and variance of the Markov jump process and matching these quantities to the drift and diffusion coefficients of an Itô stochastic differential equation (SDE). If we write $d \mathbf{X}(t)$ for the $k$-vector giving the change in state of each species in the time interval $(t, t+d t]$ then $d \mathbf{X}(t)=\mathbf{A}^{\prime} d \mathbf{R}(t)$ where $d \mathbf{R}(t)$ is the $r$-vector whose $i$ th element is a Poisson random quantity with mean $h_{i}\left(\mathbf{X}(t), c_{i}\right) d t$. Hence, we arrive at

$$
E\{d \mathbf{X}(t)\}=\mathbf{A}^{\prime} \mathbf{h}(\mathbf{X}(t), \mathbf{c}) d t, \quad \operatorname{Var}\{d \mathbf{X}(t)\}=\mathbf{A}^{\prime} \operatorname{diag}\{\mathbf{h}(\mathbf{X}(t), \mathbf{c}) d t\} \mathbf{A},
$$

where $\mathbf{h}(\mathbf{X}(t), \mathbf{c})=\left(h_{1}\left(\mathbf{X}(t), c_{1}\right), \ldots, h_{r}\left(\mathbf{X}(t), c_{r}\right)\right)^{\prime}$ is the $r$-vector of hazards. Consequently, the Itô SDE with the same infinitesimal mean and variance as the true Markov jump process is

$$
d \mathbf{X}(t)=\mathbf{A}^{\prime} \mathbf{h}(\mathbf{X}(t), \mathbf{c}) d t+\sqrt{\mathbf{A}^{\prime} \operatorname{diag}\{\mathbf{h}(\mathbf{X}(t), \mathbf{c})\} \mathbf{A}} d \mathbf{W}(t),
$$

where $d \mathbf{W}(t)$ is the increment of a $k$-dimensional Brownian motion and $\sqrt{\mathbf{A}^{\prime} \operatorname{diag}\{\mathbf{h}(\mathbf{X}(t), \mathbf{c})\} \mathbf{A}}$ is any $k \times k$ matrix square root. Note that ignoring the driving 
noise term in (1) will yield the deterministic ordinary differential equation (ODE) representation of the system. The SDE in (1) will be typically analytically intractable and it is therefore natural to work with the Euler-Maruyama approximation

$$
\Delta \mathbf{X}(t)=\mathbf{A}^{\prime} \mathbf{h}(\mathbf{X}(t), \mathbf{c}) \Delta t+\sqrt{\mathbf{A}^{\prime} \operatorname{diag}\{\mathbf{h}(\mathbf{X}(t), \mathbf{c})\} \mathbf{A}} \Delta \mathbf{W}(t)
$$

where $\Delta \mathbf{W}(t) \sim \mathrm{N}(0, \mathbf{I} \Delta t)$. Given the intractability of the CLE, we eschew this approach in favour of a further approximation which generally processes a greater degree of tractability than the CLE. This linear noise approximation (LNA) is the subject of the next section.

\subsection{Linear Noise Approximation}

The LNA can be viewed either as an approximation to the MJP or CLE and consequently can be obtained in a number of more or less formal ways. Here, we derive the LNA as a general approximation to the solution of an arbitrary SDE before considering the specific SDE given by the CLE. For further details of the LNA, we refer the reader to Komorowski et al. (2009) and Fearnhead et al. (2014) for recent discussions.

Consider now the SDE satisfied by an Itô process $\{\mathbf{X}(t)\}$ of length $k$,

$$
d \mathbf{X}(t)=\boldsymbol{\alpha}(\mathbf{X}(t)) d t+\epsilon \boldsymbol{\beta}(\mathbf{X}(t)) d \mathbf{W}(t),
$$

with initial condition $\mathbf{X}(0)=\mathbf{x}_{0}$. Let $\boldsymbol{\eta}(t)$ be the (deterministic) solution to

$$
\frac{d \boldsymbol{\eta}}{d t}=\boldsymbol{\alpha}(\boldsymbol{\eta})
$$

with initial value $\boldsymbol{\eta}_{0}$. We assume that over the time interval of interest $\|\mathbf{X}-\boldsymbol{\eta}\|$ is $O(\epsilon)$. Set $\mathbf{M}(t)=(\mathbf{X}(t)-\boldsymbol{\eta}(t)) / \epsilon$ and Taylor expand $\mathbf{X}(t)$ about $\boldsymbol{\eta}(t)$ in (3). Collecting terms of $O(\epsilon)$ gives

$$
d \mathbf{M}(t)=\mathbf{F}(t) \mathbf{M}(t) d t+\boldsymbol{\beta}(t) d \mathbf{W}(t),
$$

where $\mathbf{F}$ is the $k \times k$ matrix with components

$$
F_{i j}(t)=\left.\frac{\partial \alpha_{i}}{\partial x_{j}}\right|_{\boldsymbol{\eta}(t)} \quad \text { and } \quad \boldsymbol{\beta}(t)=\boldsymbol{\beta}(\boldsymbol{\eta}(t)) .
$$

The initial condition for (5) is $\mathbf{M}(0)=\left(\mathbf{x}_{0}-\boldsymbol{\eta}_{0}\right)$, and thereafter $\mathbf{M}(t)$ is Gaussian for all $t$, provided that the initial condition is a fixed point mass or follows a Gaussian distribution. The $\epsilon$ in (3) indicates that the intrinsic noise term $\epsilon \boldsymbol{\beta}(\mathbf{X}(t))$ is "small", but plays no part in the form of (5). For simplicity of presentation, therefore, and without loss of generality we henceforth set $\epsilon=1$.

Suppose now that $\mathbf{M}(0) \sim \mathrm{N}\left(\mathbf{m}_{0}, \mathbf{V}_{0}\right)$; in this case the SDE satisfied by $\mathbf{M}(t)$ in equation (5) can be solved analytically (see Appendix A.1) to give

$$
\mathbf{M}(t) \sim \mathrm{N}\left(\mathbf{G}(t) \mathbf{m}_{0}, \mathbf{G}(t) \mathbf{\Psi}(t) \mathbf{G}(t)^{\prime}\right)
$$

Here $\mathbf{G}$ is the fundamental matrix for the deterministic ODE $d \mathbf{m} / d t=\mathbf{F}(t) \mathbf{m}$, so that

$$
\frac{d \mathbf{G}}{d t}=\mathbf{F}(t) \mathbf{G} ; \quad \mathbf{G}(0)=\mathbf{I}
$$


and $\Psi$ satisfies

$$
\frac{d \boldsymbol{\Psi}}{d t}=\mathbf{G}^{-1}(t) \boldsymbol{\beta}(t) \boldsymbol{\beta}(t)^{\prime}\left(\mathbf{G}^{-1}(t)\right)^{\prime} ; \quad \boldsymbol{\Psi}(0)=\mathbf{V}_{0}
$$

Hence we obtain

$$
\mathbf{X}(t) \sim \mathrm{N}\left(\boldsymbol{\eta}(t)+\mathbf{G}(t) \mathbf{m}_{0}, \mathbf{G}(t) \mathbf{\Psi}(t) \mathbf{G}(t)^{\prime}\right) .
$$

In the following, we aim to exploit the analytic tractability of the LNA to build a novel hybrid model allowing both efficient simulation and inference.

\section{Hybrid Simulation via the LNA}

Hybrid simulation strategies begin by partitioning the reactions into two subsets, "fast" and "slow". It is helpful at this point to also label any species that are changed by one or more fast reactions as fast and the remaining species as slow. In between any two slow reaction events we model the dynamics of each species changed by the action of a fast reaction via the LNA. Since the slow reaction hazards will, in general, depend on species changed by fast reaction occurrences, slow reaction event times will follow an inhomogeneous Poisson process. We simulate slow reaction events via thinning (Lewis \& Shedler 1979), which requires an upper bound on the total slow reaction intensity.

In the following section, we give a novel dynamic re-partitioning scheme and provide a justification of the approach. In Section 3.2, we derive a probable bound on a linear combination of LNA components before using this result to give a probable upper bound on the total intensity of all slow reactions in Section 3.3. We describe our hybrid simulation strategy algorithmically in Section 3.4 .

\subsection{Choice of reaction type}

Consider the general criterion that over some time interval $\Delta t$ the changes brought about by reaction $j$ have a small relative impact on the state vector, $\mathbf{X}$; such changes will also have a small relative impact on the rate of each reaction. We represent a typical number of occurrences of a reaction by its expectation; however even if this expectation is less than one, we do not wish a single occurrence of $j$ to cause a substantial change in the state vector. For a reaction $j$ to be regarded as fast, we therefore require

$$
\left|a_{j i}\right| \max \left(1, h_{j} \Delta t\right) \leq \epsilon X_{i}
$$

for all $i$ such that $a_{j i} \neq 0$ and for some $\epsilon>0$ which represents "small".

Our proposed scheme re-evaluates the choice of reactions which can safely be modelled as fast at intervals of at most $\Delta t_{\text {hybrid }}$. Clearly this choice must be valid until the next re-evaluation and so, we require (9) to hold with $\Delta t_{\text {hybrid }}$ and $\epsilon$ equal to some $\epsilon_{\text {hybrid }}$.

Both the CLE and LNA are based upon the Gaussian approximation to the Poisson distribution; let us deem this approximation to be sufficiently accurate provided that the mean of the Poisson distribution is at least $N^{*}$. We therefore require that, over the time interval where changes brought about by reaction $j$ start to noticeably affect the rates of at least one reaction (which may be reaction $j$ ), the mean number of occurrences of reaction $j$ should be at least $N^{*}$. Let $\Delta t_{j}$ be the time interval over which changes brought about 
by reaction $j$ start to have an effect. Now for some suitable choice of $\epsilon=\epsilon^{*}, \Delta t_{j}$ is the largest value $\Delta t$ which satisfies (9). Clearly if $\left|a_{j i}\right|>\epsilon^{*} X_{i}$ for at least one $i$ then (9) cannot be satisfied and the reaction must be slow. Otherwise $\Delta t_{j}$ is the largest $\Delta t$ that satisfies $\left|a_{j i}\right| h_{j} \Delta t \leq \epsilon^{*} X_{i} \forall i$; i.e. $h_{j} \Delta t_{j}=\epsilon^{*} \min _{i} \frac{1}{\left|a_{j i}\right|} X_{i}$. We however need $h_{j} \Delta t_{j} \geq N^{*}$; for an equation to be considered as fast we must therefore require that

$$
\left|a_{j i}\right| N^{*} \leq \epsilon^{*} X_{i}
$$

for all $i$ such that $a_{j i} \neq 0$. As might be inferred from the italicised fundamental condition, $\Delta t_{j}$ does not appear explicitly in this equation. Note also that subject to (10), the requirement in (9) $\left|a_{j i}\right| \leq \epsilon X_{i} \forall i$ is automatically satisfied provided $\epsilon \geq \epsilon^{*} / N^{*}$.

In summary, for reaction $j$ to be classified as fast, we require (10) to be satisfied, and (9) to be satisfied for $\Delta t=\Delta t_{\text {hybrid }}$ and $\epsilon=\epsilon_{\text {hybrid }}$.

\subsection{Probable bounds on a linear combination of LNA components}

An upper bound on the total intensity of all slow reactions can be found by deriving an upper bound on a linear combination of the components that drive the LNA. We therefore require an upper bound of a function of the form $\sum_{i=1}^{k} b_{i}^{*}(t) M_{i}(r), r \in[0, t]$, where $\mathbf{M}(r)$ satisfies (5). The following result provides a bound which holds with probability as close to 1 as desired. A proof can be found in A.2.

Proposition 1 Let $M_{i}(t), i=1, \ldots, k$ be the components of the stochastic vector $\mathbf{M}(t)$ which satisfies $\mathbf{M}(0)=\mathbf{0}$ and evolves according to (5). Define

$$
\tau_{i}(t):=\int_{0}^{t} \sum_{j=1}^{k}\left[\mathbf{G}^{-1}(r) \boldsymbol{\beta}(r)\right]_{i j}^{2} d r,
$$

where $\mathbf{G}(t)$ is the deterministic matrix defined in (7). Set $\mathbf{b}(t)=\mathbf{G}(t)^{\prime} \mathbf{b}^{*}(t)$, and

$$
b_{i}^{\max }:=\max _{r \in[0, t]}\left|b_{i}(r)\right|, i=1, \ldots, k .
$$

For any $\epsilon \in(0,1)$ and every $i$ in $1, \ldots, k$ define

$$
u_{i}^{*}:=-\Phi^{-1}\left(\frac{\epsilon}{4 k}\right) \tau_{i}^{1 / 2},
$$

where $\Phi(\cdot)$ is the cumulative distribution function of a standard normal distribution. Then

$$
\mathbb{P}\left(\max _{r \in[0, t]} \sum_{i=1}^{k} b_{i}^{*}(r) M_{i}(r) \leq \sum_{i=1}^{k} b_{i}^{\max } u_{i}^{*}\right) \geq 1-\epsilon
$$

\subsection{Maximum intensity over an interval}

The evolution of species numbers that arises from fast reactions is modelled via the LNA, whereas changes in species numbers that arise from slow reactions are modelled though the Markov Jump process. In order to efficiently simulate slow reaction events we require a relatively tight upper bound on the total hazard (or intensity) of all slow reactions. 
Consider the time interval between a given slow reaction event and either the next slow reaction or the time $\left(\Delta t_{\text {hybrid }}\right.$ in the future) when reactions may be reclassified. Over this interval the number of molecules of each slow species remains fixed, with changes in reaction hazards depending only on the evolution of the relevant fast species. A first order reaction where the rate depends only on the number of molecules of a single slow species may therefore be treated, over this interval, as zeroth order, but with a different rate constant. Similarly a second order reaction where one or both of the reacting species are slow can be treated as a first or zeroth order reaction over this interval. In common with most reaction models (e.g. Wilkinson (2012)) we will assume that any apparent interactions between more than two molecules are built up from reactions of order two or fewer. For this interval we therefore partition the slow reactions into three classes $R_{s}^{(0)}$, $R_{s}^{(1)}$ and $R_{s}^{(2)}$, for reactions which, over this interval can be treated as zeroth, first and second order respectively, and where these classifications are understood to depend on the current classification of reactions into slow and fast.

Denoting by $X_{k}$ the number of molecules of species $k$, we therefore have $h_{j}\left(t, c_{j}\right)=c_{j}^{*}$ for $j \in R_{s}^{(0)} ; h_{j}\left(t, c_{j}\right)=c_{j}^{*} X_{k_{1}(j)}$ for $j \in R_{s}^{(1)}$; and $h_{j}\left(t, c_{j}\right)=c_{j}^{*} X_{k_{1}(j)} X_{k_{2}(j)}$ for $j \in R_{s}^{(2)}$, where $k_{1}(j)$ and $k_{2}(j)$ are the indices of the first and second (if required) reactants involved in reaction $j$, and each coefficient, $c_{j}^{*}$, is proportional to the true rate constant, $c_{j}$, but also takes into account the number of molecules of any slow reactants in reaction $j$.

Writing $X_{i}(t)=\eta_{i}(t)+M_{i}(t)$ and neglecting terms in $M_{i} M_{j}$, the total intensity of all slow reactions is

$$
\begin{aligned}
\lambda^{(s)}(\mathbf{X}(t)) \approx & \sum_{j \in R_{s}^{(0)}} c_{j}^{*}+\sum_{j \in R_{s}^{(1)}} c_{j}^{*}\left(\eta_{k_{1}(j)}(t)+M_{k_{1}(j)}(t)\right) \\
+ & \sum_{j \in R_{s}^{(2)}} c_{j}^{*}\left(\eta_{k_{1}(j)}(t) \eta_{k_{2}(j)}(t)+\eta_{k_{1}(j)}(t) M_{k_{2}(j)}(t)+\eta_{k_{2}(j)}(t) M_{k_{1}(j)}(t)\right) \\
= & \lambda^{(s)}(\boldsymbol{\eta}(t))+\sum_{j \in R_{s}^{(1)}} c_{j}^{*} M_{k_{1}(j)}(t) \\
& \quad+\sum_{j \in R_{s}^{(2)}} c_{j}^{*}\left(\eta_{k_{1}(j)}(t) M_{k_{2}(j)}(t)+\eta_{k_{2}(j)}(t) M_{k_{1}(j)}(t)\right)
\end{aligned}
$$

This can be rewritten as

$$
\lambda^{(s)}(X(t)) \approx \lambda^{(s)}(\boldsymbol{\eta}(t))+\sum_{i=1}^{k} b_{i}^{*}\left(\mathbf{c}^{*}, \boldsymbol{\eta}(t)\right) M_{i}(t)
$$

where

$$
b_{i}^{*}\left(\mathbf{c}^{*}, \boldsymbol{\eta}(t)\right)=\sum_{\left\{j \in R_{s}^{(1)}: k_{1}(j)=i\right\}} c_{j}^{*}+\sum_{\left\{j \in R_{s}^{(2)}: k_{2}(j)=i\right\}} c_{j}^{*} \eta_{k_{1}(j)}+\sum_{\left\{j \in R_{s}^{(2)}: k_{1}(j)=i\right\}} c_{j}^{*} \eta_{k_{2}(j)} .
$$

Note that the approximation in (14) is exact if, over the interval, all reactions can be treated as zeroth or first order. Also $b_{i}=0$ if all reactions whose rate is influenced by species $i$ can be treated as zeroth order reactions over the time interval. 
Defining $b_{i}^{\max }$ and $u_{i}^{*}$ as in $(12)$ and (13) and, given that we choose to make $M_{i}(0)=0$, we may therefore provide the following probable upper bound over the interval $[0, T]$ on the total intensity of all slow reactions combined:

$$
h_{\max }^{s}:=\lambda_{\max }^{s}+\sum_{i=1}^{k} b_{i}^{\max } u_{i}^{*}
$$

where

$$
\lambda_{\max }^{s}:=\max _{t \in[0, T]} \lambda^{s}\left(\mathbf{c}^{*}, \boldsymbol{\eta}(t)\right)
$$

\subsection{Generic Algorithm}

We now present a generic algorithm for simulating from a mixture of slow and fast reactions using the Linear Noise Approximation for the fast reactions and allowing the slow reactions to evolve through the "exact" Markov jump process.

Given a starting state the algorithm chooses a time interval, $\Delta t_{\text {integrate }}$, over which to integrate the fast reaction mechanism and hence detect whether or not there has been a potential slow reaction. If there is a potential slow reaction in this interval then the fast reactions must be reintegrated up to this potential slow reaction time to simulate the state vector at this time. If the next slow reaction were to occur some considerable time in the future then $\left[t_{\text {curr }}, t_{\text {curr }}+\Delta t_{\text {integrate }}\right]$ would ideally just fail to include this reaction time, and thereby eliminate the need to re-integrate over such a large time interval. By contrast the penalty to computational efficiency is smaller if there is just a small time interval until the next potential slow reaction. However the upper bound on the total slow intensity, and hence the rate at which potential reactions occur, increases with $\Delta t_{\text {integrate }}$. Given the circularity of these constraints we simply set $\Delta t_{\text {integrate }}$ as an arbitrary tuning factor. Furthermore, since we may only re-evaluate the fast/slow status of each reaction at the end of an integration we require $\Delta t_{\text {integrate }} \leq \Delta t_{\text {hybrid }}$.

The algorithm commences at time $t_{\text {curr }}=0$ with an initial state vector of $\mathbf{x}_{\text {curr }}:=$ $\left(x_{c u r r, 1}, \ldots, x_{c u r r, k}\right)$ and ends at some pre-defined time $t_{\text {end }}>0$ with $\mathbf{x}_{\text {curr }}$ corresponding to the the state vector at $t_{\text {end }}$. The rate constants $\mathbf{c}$ are assumed to be known but to simplify our presentation of the algorithm we remove explicit mention of $\mathbf{c}$ from the notation. The algorithm starts with $\Delta t_{\text {integrate }}$ and $\Delta t_{\text {hybrid }}$ set to their default (user-defined) values.

1. If $t_{\text {curr }} \geq t_{\text {end }}$ then stop.

2. Set $\Delta t_{\text {hybrid }}=\min \left(\Delta t_{\text {hybrid }}, t_{\text {end }}-t_{\text {curr }}\right)$ and $\Delta t_{\text {integrate }}=\min \left(\Delta t_{\text {integrate }}, t_{\text {end }}-t_{\text {curr }}\right)$.

3. Classify reactions: given $\mathbf{x}_{\text {curr }}$ classify each reaction as either slow or fast.

4. Preliminary integration over full interval: integrate jointly over $\left[t_{\text {curr }}, t_{\text {curr }}+\Delta t_{\text {integrate }}\right]$ the $k$-vector ODE for $\boldsymbol{\eta}(t)$, (4), the $k \times k$ matrix ODE for $\mathbf{G}(t)$, (7), the ODEs for $\boldsymbol{\Psi}(t)$, (8), and the integral for $\tau_{i}\left(t_{\text {curr }}, \Delta t_{\text {integrate }}\right)(i=1, \ldots, k)$, (11). Initial conditions for the ODEs are $\boldsymbol{\eta}(0)=\mathbf{x}_{\text {curr }}, \mathbf{G}(0)=\mathbf{I}$ and $\boldsymbol{\Psi}(0)=\mathbf{0}$. So that only fast reactions contribute to the evolution, for the purposes of this integration set the rate of each slow reaction to zero. 
5. Keep running maxima over the course of the ODE integration in order to calculate $\lambda_{\text {max }}^{s}$ and $b_{i}^{\max }$ over the interval $\left[t_{\text {curr }}, t_{\text {curr }}+\Delta t_{\text {integrate }}\right]$.

6. Calculate $u_{i}^{*}(i=1, \ldots, k)$ from (13).

7. Simulate the first event time $t_{*}$ from a Poisson process which starts at $t_{c u r r}$ and has intensity $h_{\max }^{s}$ as given in (16).

8. If $t_{*}>t_{\text {curr }}+\Delta t_{\text {integrate }}$ then there is no potential slow reaction in $\left[t_{\text {curr }}, t_{\text {curr }}+\right.$ $\left.\Delta t_{\text {integrate }}\right]$; set $t_{\text {curr }}=t_{\text {curr }}+\Delta t_{\text {integrate }}$ and simulate the state vector at this new time, $\mathrm{x}_{t_{c u r r}} ;$ go to Step 1 .

9. Second integration: integrate the ODEs from Step 4 (except (11)) forward over the interval $\left[t_{c u r r}, t_{*}\right)$, again with the rate of each slow reaction set to zero. This provides the distribution of of the species just before time $t_{*}, \mathbf{X}\left(t_{*}^{-}\right)$, given that no slow reactions occurred up until this time. Hence simulate $\mathbf{x}\left(t_{*}^{-}\right)$and set $\mathbf{x}_{\text {curr }} \leftarrow \mathbf{x}\left(t_{*}^{-}\right)$.

10. Calculate the probability that a slow reaction actually occurs at $t_{*}, \lambda^{s}\left(\mathbf{x}_{\text {curr }}\right) / h_{\text {max }}^{s}$, and hence simulate whether or not a slow reaction occurs at $t_{*}$.

11. If no slow reaction occurs then set $t_{c u r r}=t_{*}$ and go to Step 2 .

12. Update from slow reaction: simulate which slow reaction occurs using the following probabilities for $j \in R_{s}$.

$$
\mathbb{P}(\text { slow reaction } j \mid \text { slow reaction })=\frac{h_{j}\left(\mathbf{x}_{\text {curr }}\right)}{\lambda^{s}\left(\mathbf{x}_{\text {curr }}\right)} ;
$$

update $\mathbf{x}_{\text {curr }}$ according to the net effects vector for the chosen slow reaction.

13. Set $t_{c u r r}=t_{*}$ and go to $\operatorname{Step} 2$,

\section{Bayesian Inference}

We consider here the task of performing inference for the kinetic rate constants $\mathbf{c}$ given noisy measurements on the system state $\mathbf{X}(t)$ at discrete time points. We aim to embed the hybrid simulation method outlined in Section 3 inside a recently proposed particle MCMC algorithm to obtain an efficient inference scheme.

\subsection{A Particle MCMC approach}

Suppose that the process $\mathbf{X}(t)$ is not observed exactly, rather, we have (without loss of generality) noisy measurements $\mathbf{Y}_{0: T}=\{\mathbf{Y}(t): t=0, \ldots, T\}$ observed on a regular grid. We assume that the true underlying process $\mathbf{X}(t)$ is linked to $\mathbf{Y}(t)$ via the density $\pi(\mathbf{y}(t) \mid \mathbf{x}(t))$. Moreover, we assume that the observations are conditionally independent given the latent process.

Rather than perform inference for the exact Markov jump process, we work with the hybrid model, and kinetic rate constants $\mathbf{c}$ governing this approximate model. Let $\mathbf{X}_{(0, T]}=$ 
$\{\mathbf{X}(t): t \in(0, T]\}$ denote the complete process path on $(0, T]$ and denote the marginal density of $\mathbf{X}_{(0, T]}$, under the structure of the hybrid model, by $\pi_{h}\left(\mathbf{x}_{(0, T]} \mid \mathbf{x}(0), \mathbf{c}\right)$, since it depends on the starting value $\mathbf{x}(0)$ and the rate constants c. Note that this density can be sampled from by executing the algorithm described in Section 3 . Let $\pi(\mathbf{x}(0))$ and $\pi(\mathbf{c})$ denote the respective prior densities for $\mathbf{X}(0)$ and $\mathbf{c}$. Fully Bayesian inference may proceed by sampling

$$
\pi\left(\mathbf{c}, \mathbf{x}_{[0, T]} \mid \mathbf{y}_{0: T}\right) \propto \pi(\mathbf{c}) \pi(\mathbf{x}(0)) \pi_{h}\left(\mathbf{x}_{(0, T]} \mid \mathbf{x}(0), \mathbf{c}\right) \prod_{i=0}^{T} \pi(\mathbf{x}(i) \mid \mathbf{y}(i)) .
$$

In this work, interest lies in the marginal posterior density

$$
\begin{aligned}
\pi\left(\mathbf{c} \mid \mathbf{y}_{0: T}\right) & =\int \pi\left(\mathbf{c}, \mathbf{x}_{[0, T]} \mid \mathbf{y}_{0: T}\right) d \mathbf{x}_{[0, T]} \\
& \propto \pi(\mathbf{c}) \pi\left(\mathbf{y}_{0: T} \mid \mathbf{c}\right)
\end{aligned}
$$

Inference is problematic due to the intractability of the marginal likelihood $\pi\left(\mathbf{y}_{0: T} \mid \mathbf{c}\right)$. We generate samples (17) by appealing to a special case of the particle marginal Metropolis Hastings (PMMH) scheme described in Andrieu et al. (2010) and Andrieu, Doucet \& Holenstein (2009). In brief, we propose a new $\mathbf{c}^{*}$ using a suitable proposal kernel $q\left(\mathbf{c}^{*} \mid \mathbf{c}\right)$ and run a particle filter targeting $\pi\left(\mathbf{x}_{[0, T]} \mid \mathbf{y}_{0: T}, \mathbf{c}^{*}\right)$ to obtain the filter's estimate of marginal likelihood, denoted $\hat{\pi}\left(\mathbf{y}_{0: T} \mid \mathbf{c}^{*}\right)$. At iteration $i$ the proposed $\mathbf{c}^{*}$ is accepted with probability

$$
\min \left\{1, \frac{\hat{\pi}\left(\mathbf{y}_{0: T} \mid \mathbf{c}^{*}\right) \pi\left(\mathbf{c}^{*}\right)}{\hat{\pi}\left(\mathbf{y}_{0: T} \mid \mathbf{c}^{(i-1)}\right) \pi\left(\mathbf{c}^{(i-1)}\right)} \times \frac{q\left(\mathbf{c}^{(i-1)} \mid \mathbf{c}^{*}\right)}{q\left(\mathbf{c}^{*} \mid \mathbf{c}^{(i-1)}\right)}\right\}
$$

After initialising the rate constants and at iteration $i=0$ with $\mathbf{c}^{(0)}$, the algorithm proceeds as follows for $i \geq 1$ :

1. Draw $\mathbf{c}^{*} \sim q\left(\cdot \mid \mathbf{c}^{(i-1)}\right)$.

2. Run a particle filter targeting $\pi\left(\mathbf{x}_{[0, T]} \mid \mathbf{y}_{0: T}, \mathbf{c}^{*}\right)$, and compute $\hat{\pi}\left(\mathbf{y}_{0: T} \mid \mathbf{c}^{*}\right)$, the filter's estimate of marginal likelihood.

3. With probability (18) accept a move to $\mathbf{c}^{*}$ otherwise put $\mathbf{c}^{(i)}=\mathbf{c}^{(i-1)}$.

The scheme as presented can be seen as a pseudo-marginal Metropolis-Hastings method (Beaumont 2003, Andrieu \& Roberts 2009). In particular, provided that the estimator of marginal likelihood is non-negative and unbiased (or has a constant positive multiplicative bias that does not depend on c), it is straightforward to verify that the method targets the marginal $\pi\left(\mathbf{c} \mid \mathbf{y}_{0: T}\right)$. We let $\mathbf{u}$ denote all random variables generated by the particle filter and write the estimate of marginal likelihood as $\hat{\pi}\left(\mathbf{y}_{0: T} \mid \mathbf{c}\right)=\pi\left(\mathbf{y}_{0: T} \mid \mathbf{c}, \mathbf{u}\right)$. By augmenting the state space of the Markov chain to include $\mathbf{u}$ the acceptance ratio in (18) can be rewritten as

$$
\frac{\pi\left(\mathbf{y}_{0: T} \mid \mathbf{c}^{*}, \mathbf{u}^{*}\right) \pi\left(\mathbf{u}^{*} \mid \mathbf{c}^{*}\right) \pi\left(\mathbf{c}^{*}\right)}{\pi\left(\mathbf{y}_{0: T} \mid \mathbf{c}^{(i-1)}, \mathbf{u}^{(i-1)}\right) \pi\left(\mathbf{u}^{(i-1)} \mid \mathbf{c}^{(i-1)}\right) \pi\left(\mathbf{c}^{(i-1)}\right)} \times \frac{q\left(\mathbf{c}^{(i-1)} \mid \mathbf{c}^{*}\right) \pi\left(\mathbf{u}^{(i-1)} \mid \mathbf{c}^{(i-1)}\right)}{q\left(\mathbf{c}^{*} \mid \mathbf{c}^{(i-1)}\right) \pi\left(\mathbf{u}^{*} \mid \mathbf{c}^{*}\right)}
$$

and we see that the chain targets the joint density

$$
\pi\left(\mathbf{c}, \mathbf{u} \mid \mathbf{y}_{0: T}\right) \propto \pi\left(\mathbf{y}_{0: T} \mid \mathbf{c}, \mathbf{u}\right) \pi(\mathbf{u} \mid \mathbf{c}) \pi(\mathbf{c}) .
$$


Marginalising (19) over $\mathbf{u}$ gives $\pi\left(\mathbf{c} \mid \mathbf{y}_{0: T}\right)$ as a marginal density. We note that if interest lies in the joint posterior density of $\mathbf{c}$ and the latent path, the above algorithm can be modified to target $\pi\left(\mathbf{c}, \mathbf{x}_{[0, T]} \mid \mathbf{y}_{0: T}\right)$. Essentially, the ancestors of each particle must be stored to allow sampling of the particle filter's approximation to $\pi\left(\mathbf{x}_{[0, T]} \mid \mathbf{y}_{0: T}, \mathbf{c}^{*}\right)$. We refer the reader to Andrieu et al. (2010) for further details.

Step 2 of the PMMH scheme requires implementation of a particle filter for the successive generation of samples from $\pi\left(\mathbf{x}_{[0, j]} \mid \mathbf{y}_{0: j}, \mathbf{c}^{*}\right)$ for each $j=0,1, \ldots, T$. Note that up to proportionality, and for $j>0$

$$
\pi\left(\mathbf{x}_{[0: j]} \mid \mathbf{y}_{0: j}\right) \propto \pi(\mathbf{y}(j) \mid \mathbf{x}(j)) \pi\left(\mathbf{x}_{[0: j-1]} \mid \mathbf{y}_{0: j-1}\right) \pi_{h}\left(\mathbf{x}_{(j-1, j]} \mid \mathbf{x}(j-1)\right)
$$

where we have dropped $\mathbf{c}^{*}$ from the notation. Now suppose that we have an equally weighted sample of points (or particles) of size $N$ from $\pi\left(\mathbf{x}_{[0: j-1]} \mid \mathbf{y}_{0: j-1}\right)$. Denote this sample by $\left\{\mathbf{x}_{[0: j-1]}^{k}, k=1, \ldots, N\right\}$. The bootstrap particle filter of Gordon et al. (1993) generates an approximate sample from $\pi\left(\mathbf{x}_{[0: j]} \mid \mathbf{y}_{0: j}\right)$ with the following importance resampling algorithm:

1. For $k=1,2, \ldots, N$, draw $\mathbf{x}_{(j-1, j]}^{k} \sim \pi_{h}\left(\cdot \mid \mathbf{x}(j-1)^{k}\right)$ using the hybrid simulator and construct the extended path, $\mathbf{x}_{[0, j]}^{k}=\left(\mathbf{x}_{[0, j-1]}, \mathbf{x}_{(j-1, j]}\right)$.

2. Construct and normalise the weights,

$$
w_{k}^{(j)}=\pi\left(\mathbf{y}(j) \mid \mathbf{x}(j)^{k}\right), \quad \tilde{w}_{k}^{(j)}=\frac{w_{k}^{(j)}}{\sum_{l=1}^{N} w_{l}^{(j)}},
$$

where $k=1,2, \ldots, N$.

3. Resample $N$ times amongst the $\mathbf{x}_{[0, j]}^{k}$ using the normalised weights as probabilities.

In the case $j=0, \pi(\mathbf{x}(0) \mid \mathbf{y}(0))$ can be sampled by replacing Step 1 in the algorithm above with $N$ iid draws from the prior $\pi(\mathbf{x}(0))$. Hence, after initialising the particle filter with a sample from the prior, the above sequence of steps can be performed as each observation becomes available, with the posterior sample at one time point used as the prior for the next. By using the hybrid simulator to generate proposals inside the importance resampler, evaluation of the associated likelihood is not required when calculating the importance weights and the only term that needs to be evaluated is the tractable density associated with the measurement error. This setup is flexible and can be used with any forward simulator such as the Gillespie algorithm or chemical Langevin equation.

After all data points have been assimilated, the filter's estimate of the marginal likelihood is

$$
\hat{\pi}\left(\mathbf{y}_{0: T}\right)=\hat{\pi}(\mathbf{y}(0)) \prod_{j=0}^{T-1} \hat{\pi}\left(\mathbf{y}(j+1) \mid \mathbf{y}_{0: j}\right)=\prod_{j=0}^{T} \frac{1}{N} \sum_{k=1}^{N} w_{k}^{(j)}
$$

for which we obtain unbiasedness under mild conditions involving the resampling scheme, satisfied by the bootstrap filter described above (Del Moral 2004). Note that for the special case of the PMMH algorithm used here, when running the particle filter, we need only store the values of the latent states at each observation time, and each unnormalised weight. 


\subsubsection{Tuning}

The PMMH scheme requires specification of a number of particles $N$ to be used in the particle filter at Step 2. As noted by (Andrieu \& Roberts 2009), the mixing efficiency of the PMMH scheme decreases as the variance of the estimated marginal likelihood increases. This problem can be alleviated at the expense of greater computational cost by increasing $N$. This therefore suggests an optimal value of $N$ and finding this choice is the subject of Pitt, dos Santos Silva, Giordani \& Kohn (2012), Doucet, Pitt \& Kohn (2013) and Sherlock, Thiery, Roberts \& Rosenthal (2013). The latter suggest that $N$ should be chosen so that the variance in the noise in the estimated log-posterior is around 2. Pitt et al. (2012) note that the penalty is small for a value between 0.25 and 2.25. We therefore recommend performing an initial pilot run of daPMMH to obtain an estimate of the posterior mean for the parameters $\mathbf{c}$, denoted $\hat{\mathbf{c}}$. The value of $N$ should then be chosen so that $\operatorname{Var}\left(\log \pi\left(\mathbf{y}_{0: T} \mid \hat{\mathbf{c}}\right)\right)$ is around 2 .

In our application, we note that the rate constants $\mathbf{c}$ must be strictly positive and we update $\log (\mathbf{c})=\left(\log \left(c_{1}\right), \ldots, \log \left(c_{r}\right)\right)^{\prime}$ in a single block using a random walk proposal with Gaussian innovations. The innovation variance must be chosen appropriately to maximise statistical efficiency through well mixing chains. We take the innovation variance to be $\gamma \operatorname{vâr}(\mathbf{c})$, where vâr(c) is obtained from a short pilot run of the scheme. Following Sherlock et al. (2013) we tune the scaling parameter $\gamma$ to give an acceptance rate of approximately $10 \%$.

\section{Application: Autoregulatory Network}

To assess the performance of the proposed hybrid approach as a simulator and as an inferential model, we consider a simple autoregulatory network with two species, $\mathcal{X}_{1}$ and $\mathcal{X}_{2}$ whose time course behaviour evolves according to the following set of coupled reactions,

$$
\begin{aligned}
R_{1}: & \emptyset \stackrel{c_{1}}{\longrightarrow} \mathcal{X}_{1} & R_{2}: & \emptyset \stackrel{c_{2}}{\longrightarrow} \mathcal{X}_{2} \\
R_{3}: & \mathcal{X}_{1} \stackrel{c_{3}}{\longrightarrow} \emptyset & R_{4}: & \mathcal{X}_{2} \stackrel{c_{4}}{\longrightarrow} \emptyset \\
R_{5}: & \mathcal{X}_{1}+\mathcal{X}_{2} \stackrel{c_{5}}{\longrightarrow} 2 \mathcal{X}_{2} & &
\end{aligned}
$$

Essentially, reactions $R_{1}$ and $R_{2}$ represent immigration, reactions $R_{3}$ and $R_{4}$ represent death and finally $R_{5}$ can be thought of as interaction between the two species. Note that even for this simple system, the transition density associated with the resulting Markov jump process (under an assumption of mass action kinetics) cannot be found in closed form.

Throughout this section we take

$$
\mathbf{c}=(2, s c, 1 / 50,1,1 /(50 \times s c))^{\prime},
$$

and investigate the performance of our hybrid algorithm (henceforth designated as Hybrid $L N A$ ) with regard to both the simulated distribution of $X_{1}$ and $X_{2}$ and inference on $\mathbf{c}$ for $s c \in\{1,10,100,1000\}$. The 'probable upper bound' of Section 3.2 is fixed to hold with probability $1-10^{-6}$, whilst the relative and absolute errors of the stiff ODE solver were set to $10^{-4}$. 
We use the dynamic repartitioning procedure described in Section 3.1 with $N^{*}=15$ and $\epsilon^{*}=\epsilon=0.25$. Reactions are reclassified as fast or slow every $\Delta t_{\text {hybrid }}=\Delta t_{\text {integrate }}=0.1$ time units. For this specification, Equation (10) ensures that a reaction will be regarded as slow if the species numbers of species affected by that reaction are 60 or fewer. The rates in (21) lead to an equilibrium for the MRE of

$$
\left[X_{1}, X_{2}\right]=\left[50\left(1+s c-\sqrt{1+s c^{2}}\right), 1+\sqrt{1+s c^{2}}\right]
$$

which, for $s c \gg 1$ is approximately $[50-25 / s c, s c]$. Thus, for $s c \gg 1$, when the system is at equilibrium, $X_{1}$ is typically small, $X_{2}$ is typically large, and reactions $R_{2}$ and $R_{4}$ are typically fast.

If $R_{2}$ and $R_{4}$ were always the only fast reactions and $\mathcal{X}_{2}$ were always the only fast species then the LNA for the evolution of $X_{2}$ conditional on no slow reactions taking place would be analytically tractable and, further, there would be no need for dynamic repartitioning. We, however, do not take advantage of this special case as we wish to show the generic applicability of our method. To this end we also start each system away from equilibrium, at $\mathbf{X}(0)=(0,0)^{\prime}$.

For comparison, we also ran the Gillespie algorithm and a discrete/SDE hybrid simulation method in the spirit of the next reaction hybrid algorithm of Salis \& Kaznessis (2005) (henceforth designated as Hybrid $S D E$ ). Full details of this approach can be found in Appendix A.3. For Hybrid $S D E$ we used the same dynamic partitioning criteria and additionally specified the required Euler time step to be $\Delta t_{\text {Euler }}=0.005$, which gave an accuracy comparable with that of Hybrid LNA.

\subsection{Simulation}

Using the autoregulatory network as a test case, we ran each hybrid simulator and the Gillespie algorithm for 20,000 iterations.

Figure 1 summarises the output of each simulation procedure, for species $\mathcal{X}_{1}$ and Figure 2 shows the CPU time of each simulator, averaged over 1000 realisations (and using a much larger set of values for $s c$. We see little difference between simulator output. However, when taking into account computational cost, the advantage of either hybrid approach over the Gillespie algorithm is clear. For $s c<500$, reaction events occur relatively infrequently and the computational cost of the hybrid algorithms is dominated by the computational overhead of dynamic repartitioning. However for $s c>500$, the cost of both hybrid schemes is roughly constant, whereas the cost of the Gillespie algorithm increases linearly with $s c$. Hybrid LNA requires minimal tuning, since the LNA solution involves solving a set of ODEs, for which stiff solvers that automatically and adaptively choose the time step so as to maintain a given level of accuracy are readily available. Hybrid SDE, however, requires the user to choose a fixed Euler time-step, $\Delta t_{\text {Euler }}$, and manually attempt to balance accuracy against computational effort; moreover, since the CLE is stiff and non-deterministic, there is the possibility that any fixed $\Delta t_{\text {Euler }}$ might not maintain a desired level of accuracy throughout repeated simulations, especially with different rate constants, c. Furthermore, the slow reaction updating procedure of Hybrid SDE can be inefficient in a number of ways. The algorithm requires that only one slow reaction event occurs in the interval over which the fast species are integrated. If more than one slow reaction is detected, $\Delta t_{\text {hybrid }}$ is 


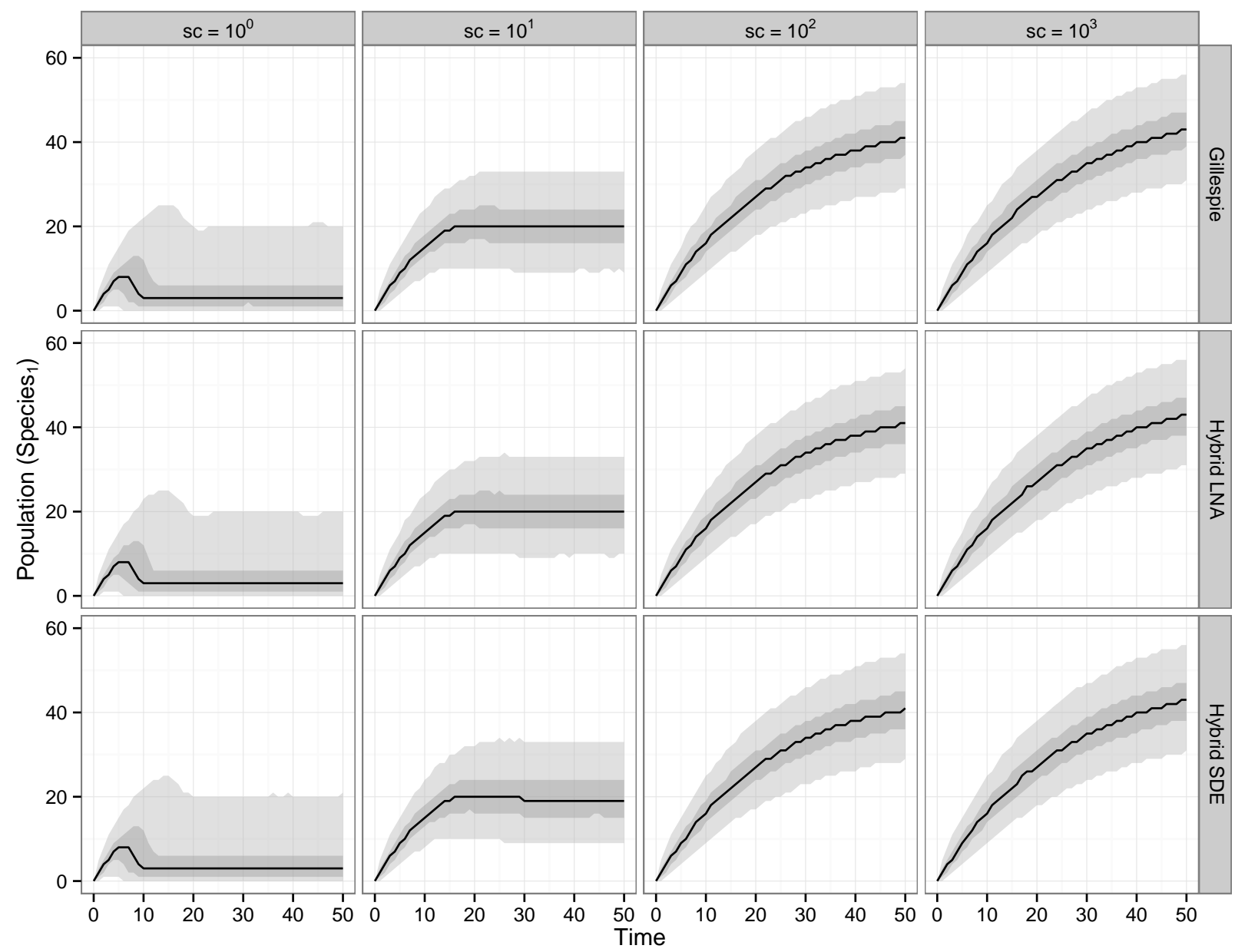

Figure 1: Median (solid), inter-quartile range (inner shaded region) and $95 \%$ credible region (outer shaded region) of $X_{1, t}$ based on 20,000 stochastic realisations of the model using Gillespie's direct method, Hybrid LNA and the Hybrid SDE. Model parameters were $(2, s c, 1 / 50,1,1 /(50 \times s c))^{\prime}$.

reduced, the system state is rewound and a reclassification of reactions takes place. Because of the reduction in $\Delta t_{\text {hybrid }}$, the system rewind may reclassify some erstwhile fast reactions as slow and so actually increase the chance of multiple slow reaction occurrences. Moreover, there is a subtle error in the algorithm: if a rewind has occurred, the new forward simulation must be conditional on the previously-simulated values of the fast reactants over the old interval of length $\Delta t_{\text {hybrid }}$. Strictly speaking therefore, these values should be stored and re-used, with approximate bridges constructed if it is necessary to fill in between the stored values. However if some of the previously-fast reactants have now become slow then it is not at all clear how to condition on the results from the previous attempt at forwards simulation. We therefore did not make make any attempt to correct this problem.

\subsection{Inference}

Data were simulated at integer times on $[0,50]$ via the Gillespie algorithm. This gave four synthetic datasets which were then corrupted to give observations with a conditional 


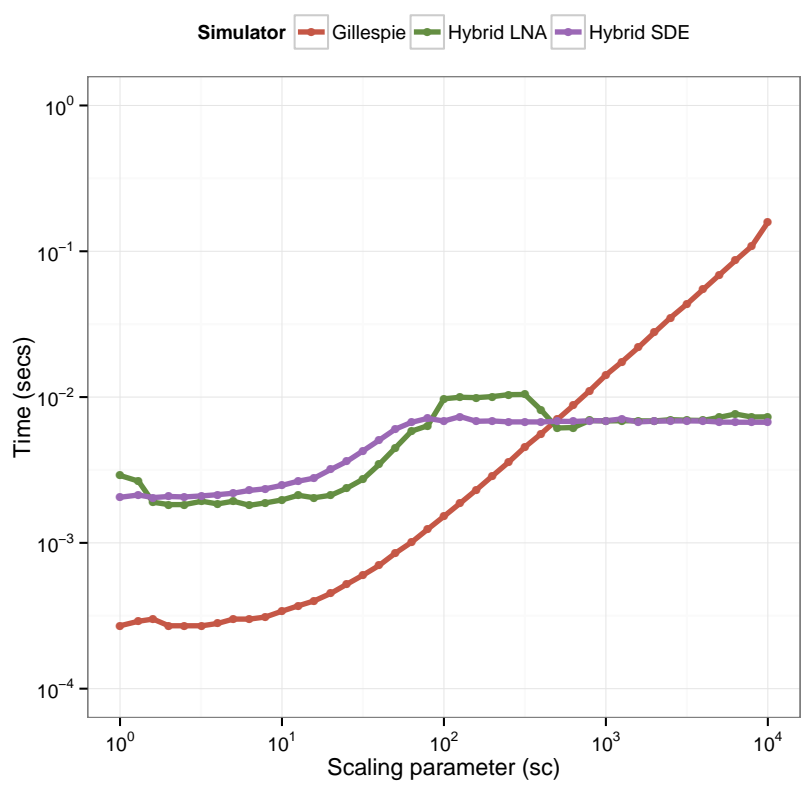

Figure 2: Simulator CPU time. Each point is the simulation time (in secs) of a single stochastic simulation, averaged over 1000 simulations. Model parameters were $(2, s c, 1 / 50,1,1 /(50 \times s c))^{\prime}$.

distribution of

$$
Y_{i}(t) \mid X_{i}(t) \sim \begin{cases}\operatorname{Poisson}\left(X_{i}(t)\right) & \text { if } X_{i}(t)>0 \\ \operatorname{Bernouilli}(0.1) & \text { if } X_{i}(t)=0\end{cases}
$$

for each component $i=1,2$. The data are plotted in Figure 3, wherein, and for the remainder of this section, we refer to the PMMH scheme that uses a given simulator by using the name of that simulator: Hybrid LNA, Hybrid SDE and Gillepsie.

To ensure identifiability, $c_{3}$ was fixed at its true value, while independent Uniform $U(-8,8)$ priors were used for the remaining $\log \left(c_{i}\right)$. For each combination of synthetic dataset and scheme we performed a pilot run with 50 particles to obtain an approximate covariance matrix $\hat{\operatorname{Var}}(\mathbf{c})$ and approximate posterior mean $\hat{\mathbf{c}}$. Following the practical advice of Sherlock et al. (2013), further pilot runs were performed with $\mathbf{c}$ fixed at $\hat{\mathbf{c}}$ to determine the number of particles $N$ that gave a variance of the estimator of $\log$-posterior $\log \pi\left(\mathbf{y}_{0: T} \mid \hat{\mathbf{c}}\right)$ of around 2. Table 1 shows the number of particles used for each scheme and each dataset. Note that Hybrid SDE required more particles than Hybrid LNA or Gillespie, with nearly an order of magnitude difference when $s c=1$. We found that using fewer particles would result in particle degeneracy around time point 32 , with only a few particles able to capture the increase in $R_{5}$ occurrences around this time point.

We performed $2 \times 10^{5}$ iterations of each scheme for $s c=1,10,100$ and $2 \times 10^{6}$ iterations for $s c=1000$. In all cases, the $\log \left(c_{i}\right)$ were updated in a single block using a Gaussian random walk proposal kernel with an innovation variance matrix given by $\gamma \hat{\operatorname{Var}}(\mathbf{c})$, with $\gamma$ tuned to give an acceptance rate of around 10\%. Figure 4 summarises the posterior output of each scheme. We see that in general, the sampled parameter values are consistent with the true values that produced the data. There appears to be little difference between the output of the PMMH scheme when using the Gillespie simulator, and both hybrid 


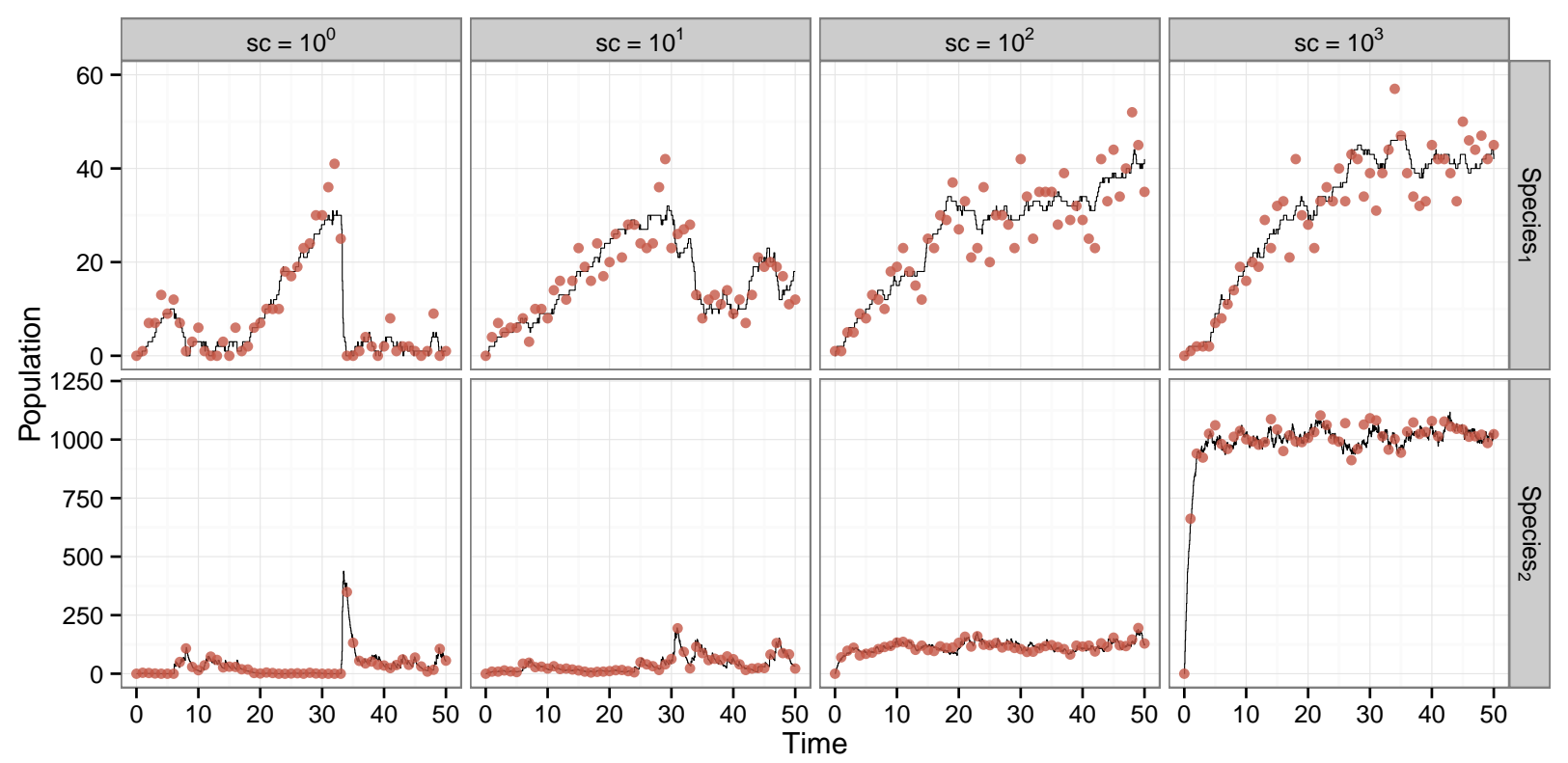

Figure 3: The four synthetic datasets used. Each data set was generated via the Gillespie algorithm. The true species numbers are represented by a black line. The noised observations are indicated by dots.

\begin{tabular}{llll}
\hline & \multicolumn{3}{c}{ Simulator } \\
\cline { 2 - 4 } sc & Gillespie & Hybrid $_{\text {LNA }}$ & Hybrid $_{\text {SDE }}$ \\
\hline $10^{0}$ & 250 & 250 & 1750 \\
$10^{1}$ & 800 & 800 & 1500 \\
$10^{2}$ & 65 & 65 & 125 \\
$10^{3}$ & 65 & 65 & 85 \\
\hline
\end{tabular}

Table 1: Number of particles used for each scheme and each synthetic dataset.

schemes, suggesting that little is lost by adopting a hybrid model to perform inference for the autoregulatory network. Figure 5 shows minimum effective sample size (ESS) per second for each scheme. The results are consistent with the timings shown in Figure 2. For relatively small values of $s c$, reaction events occur relatively infrequently and little is to be gained by running Hybrid SDE or Hybrid LNA over Gillespie. When using $s c=1000$ we see a gain in overall efficiency for the hybrid schemes. We would expect this relative gain to increase with $s c$, however, we found that the computational cost of running the PMMH scheme with the Gillespie simulator precluded comparison under this scenario.

\section{Discussion}

We have proposed a novel hybrid simulation method for efficiently simulating stochastic kinetic models (SKMs). Our approach models fast reaction dynamics with the LNA and slow dynamics with a Markov jump process. By deriving a probable upper bound for a 

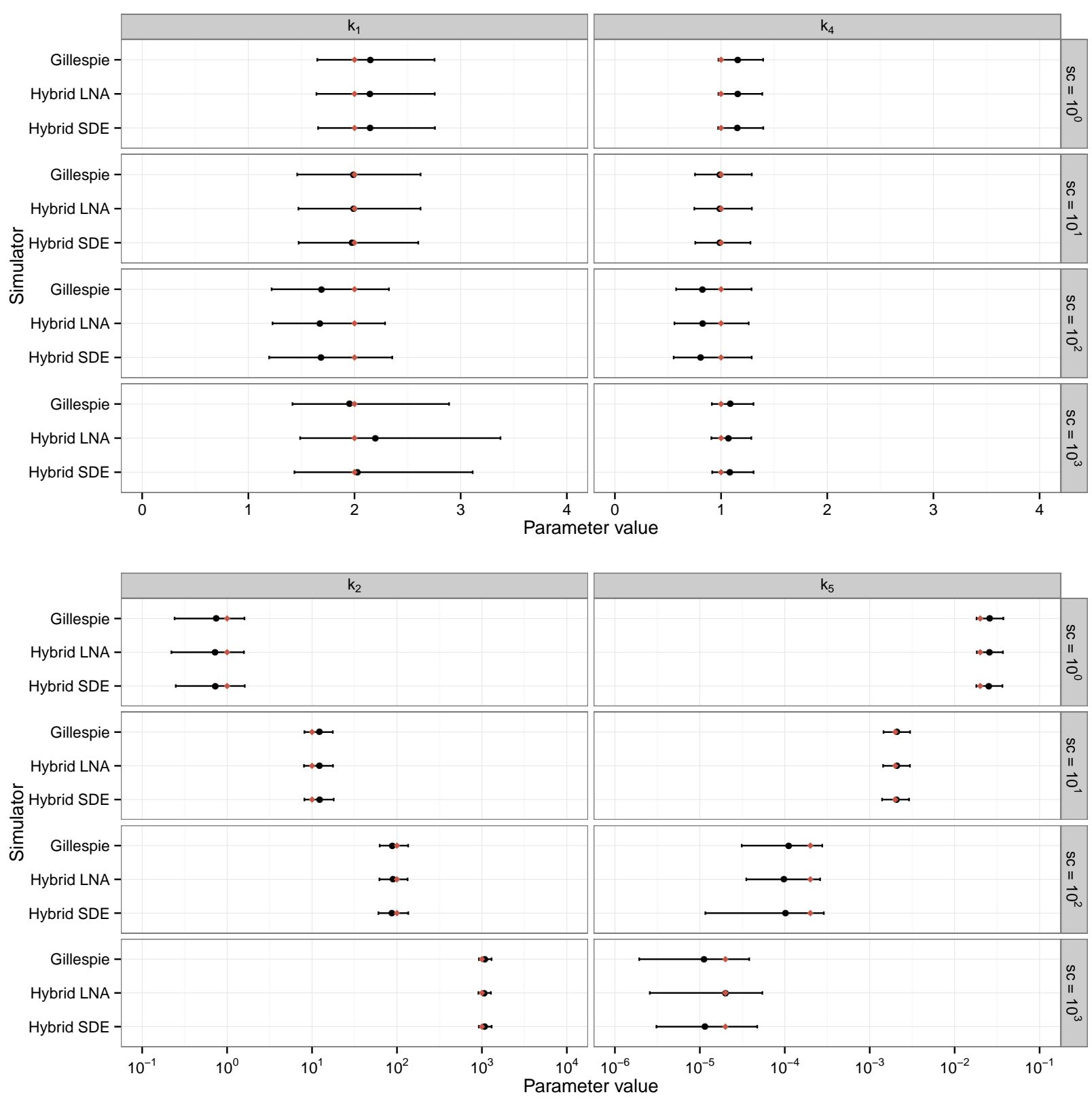

Figure 4: 95\% credible regions and posterior medians (black dot) for each parameter value based on the output of each PMCMC scheme (Gillespie, Hybrid LNA and Hybrid $\mathrm{SDE})$. True values are indicated by a red dot.

combination of components that drive the LNA, we obtain a probable upper bound for the total slow reaction hazard thus allowing exact simulation of the slow reaction events. This exactness is conditional on the accuracy of the upper bound, of the LNA approximation and of the ODE solver used to integrate the LNA. The first and the last of these were set to high values, whilst the LNA itself is expected to be accurate since it is only applied to reactions that are classified as fast. To this end, reliable criteria for the (dynamic) partitioning of reactions were also provided. Unlike existing approaches to hybrid simulation that use the CLE, we avoid the need for a system rewind (and the consequent difficulty in making the 

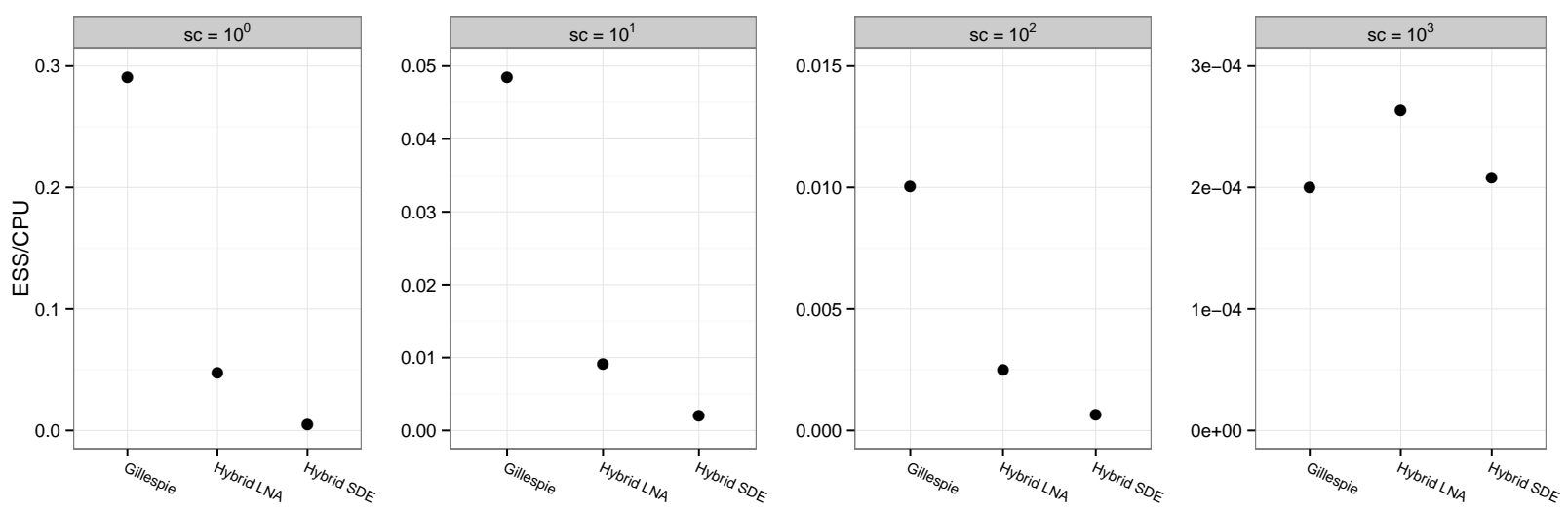

Figure 5: Minimum effective sample size (ESS) per second.

algorithm strictly correct). We also avoid the requirement to specify a fixed Euler time step which is unlikely to be appropriate across all possible sets of rate parameters with prior support and all possible realisations of the process.

We have also considered the task of inferring the rate constants governing SKMs by adopting the hybrid model and performing exact simulation-based Bayesian inference. We employed a recently-proposed particle MCMC scheme that, in its simplest implementation, only requires the ability to forward simulate from the model and evaluate an observation (or measurement error) density. We used this scheme to compare results based on our proposed hybrid simulator with those obtained under a hybrid simulator in the spirit of the work by Salis \& Kaznessis (2005), and also with inferences obtained under the "exact" Markov jump process representation of the SKM. Both hybrid schemes led to inferences that were almost indistinguishable from those under the true model, with a clear indication of increasing relative efficiency as reaction rates increased.

\section{Computing details}

All simulations were performed on a machine with 8GB of RAM and with an Intel i7 CPU. The operating system used was Ubuntu 12.04. The simulation code was mainly written in $\mathrm{C}$ and compiled with flags: -Wall, -03, -DHAVE_INLINE and -DGSL_RANGE_CHECK_OFF. FORTAN code for the stiff ODE solver came from the lsoda package (Petzold 1983). Graphics were constructed using $\mathrm{R}$ and the ggplot2 $\mathrm{R}$ package ( $\mathrm{R}$ Core Team 2013, Wickham 2009).

The code can be downloaded from

https://github.com/csgillespie/hybrid-pmcmc 


\section{A. Appendices}

\section{A.1. Solution to the LNA}

Recall that $\mathbf{G}$ is the fundamental matrix for the deterministic ODE $d \mathbf{m} / d t=\mathbf{F}(t) \mathbf{m}$, satisfying equation (7). Note that

$$
\mathbf{0}=\frac{d}{d t} \mathbf{G G}^{-1}=\mathbf{G} \frac{d \mathbf{G}^{-1}}{d t}+\frac{d \mathbf{G}}{d t} \mathbf{G}^{-1}, \text { so } \frac{d \mathbf{G}^{-1}}{d t}=-\mathbf{G}^{-1} \mathbf{F}(t)
$$

Set

$$
\mathbf{U}(t):=\mathbf{G}^{-1}(t) \mathbf{M}(t), \quad \text { so } \mathbf{U}(0)=\mathbf{M}(0) .
$$

Since $\mathbf{G}$ is deterministic, $d \mathbf{G}^{-1} d \mathbf{M}=\mathbf{0}$ and so by (5)

$$
d \mathbf{U}(t)=\mathbf{G}^{-1} \mathbf{F M} d t+\mathbf{G}^{-1} \boldsymbol{\beta} d \mathbf{W}_{t}-\mathbf{G}^{-1} \mathbf{F} \mathbf{M} d t=\mathbf{G}^{-1} \boldsymbol{\beta} d \mathbf{W}(t) .
$$

Thus

$$
\mathbf{U}(t)-\mathbf{U}(0)=\int_{0}^{t} \mathbf{G}^{-1}(r) \boldsymbol{\beta}(r) d \mathbf{W}(r) .
$$

Therefore by linearity and Ito's Isometry,

$$
\mathbf{U}(t)-\mathbf{U}(0) \sim N\left(\mathbf{0}, \int_{0}^{t} \mathbf{G}^{-1}(r) \boldsymbol{\beta}(r) \boldsymbol{\beta}(r)^{\prime}\left(\mathbf{G}^{-1}(r)\right)^{\prime} d r\right) .
$$

Suppose now that $\mathbf{M}(0)(=\mathbf{U}(0)) \sim \mathrm{N}\left(\mathbf{m}_{0}, \mathbf{V}_{0}\right)$, then

$$
\begin{aligned}
\mathbf{M}(t) & \sim N\left(\mathbf{G}(t) \mathbf{m}_{0}, \mathbf{G}(t) \mathbf{\Psi}(t) \mathbf{G}(t)^{\prime}\right) \\
\text { where } \quad \boldsymbol{\Psi}(t) & =\mathbf{V}_{0}+\int_{0}^{t} \mathbf{G}^{-1}(r) \boldsymbol{\beta}(r) \boldsymbol{\beta}(r)^{\prime}\left(\mathbf{G}^{-1}(r)\right)^{\prime} d r .
\end{aligned}
$$

\section{A.2. Proof of Proposition 1}

Firstly, $\sum_{i=1}^{k} b_{i}^{*}(r) M_{i}(r)=\sum_{i=1}^{k} b_{i}(r) U_{i}(r)$, where $U_{i}$ is the $i^{\text {th }}$ component of the vector $\mathbf{U}$ defined in Appendix A.1, but with $\mathbf{U}(0)=\mathbf{0}$ (since $\mathbf{M}(0)=\mathbf{0}$ ). From its definition, (11), $\tau_{i}$ is the $i^{\text {th }}$ diagonal component of the variance in 22 , , so

$$
\mathbb{P}\left(U_{i}(t) \geq u_{i}^{*}\right)=\Phi\left(-u_{i}^{*} / \sqrt{\tau_{i}}\right),
$$

for currently arbitrary values $u_{i}^{*}>0, i \in\{1, \ldots, k\}$.

Next, define the first hitting time $T_{i}\left(u_{i}^{*}\right)=\inf \left\{t: U_{i}(t) \geq u_{i}^{*}\right\}$. Now $U_{i}(t) \geq u_{i}^{*} \Leftrightarrow$ $T_{i}\left(u_{i}^{*}\right) \leq t=0$ so

$$
\mathbb{P}\left(U_{i}(t) \geq u_{i}^{*}\right)=\mathbb{P}\left(U_{i}(t) \geq u_{i}^{*} \mid T_{i}\left(u_{i}^{*}\right) \leq t\right) \mathbb{P}\left(T_{i}\left(u_{i}^{*}\right) \leq t\right) .
$$

By the almost sure continuity of $U_{i}, \mathbb{P}\left(U_{i}\left(T_{i}\left(u_{i}^{*}\right)\right)=u_{i}^{*}\right)=1$ and so by the symmetry of $U_{i}$, $\mathbb{P}\left(U_{i}(t) \geq u_{i}^{*} \mid T_{i}\left(u_{i}^{*}\right) \leq t\right)=1 / 2$. However $T_{i}\left(u_{i}^{*}\right) \leq t \Leftrightarrow \max _{(0, t]} U_{i} \geq u_{i}^{*}$, so

$$
\mathbb{P}\left(\max _{(0, t]} U_{i} \geq u_{i}^{*}\right)=2 \Phi\left(-u_{i}^{*} / \sqrt{\tau_{i}}\right) .
$$


Given some $\epsilon>0$, we may therefore choose $u_{i}^{*}=-\Phi^{-1}(\epsilon / 4 k) \tau_{i}^{1 / 2}$, which gives, marginally,

$$
\mathbb{P}\left(\max _{(0, t]} U_{i} \geq u_{i}^{*}\right)=\frac{\epsilon}{2 k} .
$$

By symmetry and the inclusion exclusion formula, therefore, marginally,

$$
\mathbb{P}\left(\max _{(0, t]}\left|U_{i}\right| \geq u_{i}^{*}\right)=\frac{\epsilon}{k}
$$

Hence

$$
\mathbb{P}\left(\left|U_{i}(r)\right| \leq u_{i}^{*}: i \in\{1, \ldots, k\}, r \in(0, t]\right)=1-\mathbb{P}\left(\max _{(0, t]}\left|U_{i}\right| \geq u_{i}^{*} \text { for any } i\right) \geq 1-\epsilon .
$$

Thus with probability at least $1-\epsilon$, for all $r \in[0, t]$

$$
\sum_{i=1}^{k} b_{i}^{*}(r) M_{i}(r)=\sum_{i=1}^{k} b_{i}(r) U_{i}(r) \leq \sum_{i=1}^{k}\left|b_{i}(r)\right| u_{i}^{*} \leq \sum_{i=1}^{k} b_{i}^{\max } u_{i}^{*}
$$

\section{A.3. Hybrid Simulation based on the CLE}

We consider a hybrid simulation algorithm in the spirit of the next reaction hybrid algorithm of Salis \& Kaznessis (2005). This approach treats the subset of fast species with the chemical Langevin equation and simulates their dynamics by numerically integrating the corresponding SDE. Let $\mathbf{X}^{f}(t)$ be the state of the fast species at time $t$. Suppose that we have $r^{f}$ fast reactions and $r^{s}$ slow reactions. We then arrive at

$$
d \mathbf{X}^{f}(t)=\mathbf{A}_{f}^{\prime} \mathbf{h}(\mathbf{X}(t), \mathbf{c}) d t+\sqrt{\mathbf{A}_{f}^{\prime} \operatorname{diag}\left\{\mathbf{h}^{f}(\mathbf{X}(t), \mathbf{c})\right\} \mathbf{A}_{f}} d \mathbf{W}(t)
$$

where $\mathbf{A}_{f}$ is the $r^{f} \times k^{f}$ net effect matrix associated with the fast reactions and $\mathbf{h}^{f}(\mathbf{X}(t), \mathbf{c})$ is the $r^{f}$-vector of fast reaction hazards which may depend on both fast and slow species numbers. Hence, the fast specie numbers can be simulated by recursively iterating the Euler discretisation of 23.

It remains that we can sample the times of the slow reactions. This step can be performed by Monte Carlo, equating the integral of the time dependent probability density for the time of the $j$ th slow reaction to a uniform random number. Since the slow reaction hazards are time varying, we write them as $h_{j}^{s}(t, \mathbf{c}), j=1, \ldots, r^{s}$. Let $p_{j}\left(\tau_{j} ; t_{0}\right)$ denote the next reaction probability density for the $j$ th slow reaction. Here, $t_{0}$ is the time that the last occurred and $\tau_{j}$ is the time of the $j$ th slow reaction. From Gibson \& Bruck $(2000), p_{j}\left(\tau_{j} ; t_{0}\right)$ is a time dependent exponential density for which the cumulative density function is

$$
F\left(\tau_{j} ; t_{0}\right)=1-\exp \left(-\int_{t_{0}}^{t_{0}+\tau_{j}} h_{j}^{s}\left(t^{\prime}, \mathbf{c}\right) d t^{\prime}\right) .
$$

Hence, setting equation (24) equal to a uniform random number $r_{j}$ on $(0,1)$ and simplifying gives

$$
\int_{t_{0}}^{t_{0}+\tau_{j}} h_{j}^{s}\left(t^{\prime}, \mathbf{c}\right) d t^{\prime}+\log \left(r_{j}\right)=0
$$


We solve equation (25) by rearranging it in terms of a residual $R_{j}(t)$ and setting the integral upper bound to be a variable so that

$$
\int_{t_{0}}^{t_{0}+t} h_{j}^{s}\left(t^{\prime}, \mathbf{c}\right) d t^{\prime}+\log \left(r_{j}\right)=R_{j}(t) .
$$

Plainly, if $R_{j}(t)=0$ then $t=\tau_{j}, R_{j}(t)<0$ implies that $t<\tau_{j}$ and similarly if $R_{j}(t)>0$ then $t>\tau_{j}$. Hence, starting with state $\mathbf{X}(t)$ at time $t$, we can compute $\mathbf{X}(t+\Delta t)$ assuming no slow reaction has occurred in $\left(t, t+\Delta t\right.$ ]. If the residual $R_{j}(t)$ has performed a zero crossing in $(t, t+\Delta t]$ then the $j$ th slow reaction has occurred. We monitor $R_{j}(t)$ by writing equation (26) in differential form,

$$
\frac{d R_{j}(t)}{d t}=h_{j}^{s}(t, \mathbf{c}), \quad R_{j}\left(t_{0}\right)=\log \left(r_{j}\right) .
$$

Equation (27) can then be solved by using a time discretisation method such as the Euler scheme. Note that the method is restricted to only one slow reaction event in $(t, t+\Delta t]$. If more than one zero crossing occurs in this interval then $\Delta t$ can be reduced, and the state restored to the previous one. Hence, if the $j$ slow reaction occurs, the reaction time $\tau_{j}$ can be found through an Itô-Taylor series expansion of (27). If $t^{\prime}$ is the time just prior to the $j$ th slow reaction then

$$
\tau_{j}=-\frac{R_{j}\left(t^{\prime}\right)}{h_{j}^{s}\left(t^{\prime}, \mathbf{c}\right)}+t^{\prime}
$$

The scheme provides an accurate way of capturing a slow reaction event provided that over the interval of interest, say $\left[t_{c u r r}, t_{c u r r}+\Delta t_{\text {integrate }}\right]$, it is known that only one reaction occurs. Consequently, if more than one zero crossing is recorded, the interval length is reduced until at most one slow event is captured.

The algorithm commences at time $t_{c u r r}=0$ with known rate constants $\mathbf{c}$, a known number molecules $\mathbf{x}_{\text {curr }}$ and $R_{j}(0)=\log \left(r_{j}\right), j=1, \ldots, r^{s}$. The algorithm ends with $\mathbf{x}_{\text {curr }}$ as the state vector at time $t_{\text {end }}>t_{\text {curr }}$. For simplicity, we take the length of the time interval over which a slow reaction is detected to be $\Delta t_{\text {integrate }}=\Delta t_{\text {hybrid }}$.

1. If $t_{\text {curr }} \geq t_{\text {end }}$ then stop.

2. Set $\Delta t_{\text {hybrid }}=\min \left(\Delta t_{\text {hybrid }}, t_{\text {end }}-t_{\text {curr }}\right)$.

3. Classify reactions: given $\mathbf{x}_{\text {curr }}$ classify each reaction as either slow or fast.

4. Calculate the fast reaction hazards. Using an Euler time step of $\Delta t_{\text {euler }}$, numerically integrate the SDE (23) for the fast species over $\left(t_{\text {curr }}, t_{\text {curr }}+\Delta t_{\text {hybrid }}\right.$ ] giving a sample path for the fast species over $\left(t_{\text {curr }}, t_{c u r r}+\Delta t_{\text {hybrid }}\right]$.

5. Using the slow reaction hazards, compute each residual $R_{j}(t), j=1, \ldots, r^{s}$ using an Euler approximation of (27) and decide whether or not a slow reaction has happened in $\left(t_{\text {curr }}, t_{\text {curr }}+\Delta t_{\text {hybrid }}\right]$.

6. If no slow reaction has occurred, set $t_{c u r r}:=t_{c u r r}+\Delta t_{\text {hybrid }}$ and update the fast species to their proposed values at $t_{c u r r}$; go to Step 1. 
7. If one slow reaction has occurred, identify the type $j$ and time $\tau_{j}$, set $t_{c u r r}=\tau_{j}$ and update the system to $\tau_{j}$ using the same random numbers as in step (d). Reset the $j$ th residual, $R_{j}(t)=\log \left(r_{j}\right)$. Reset $\Delta t_{\text {hybrid }}$ to its initial value if required. Goto Step 1.

8. If more than one slow reaction has occurred, reduce $\Delta t_{\text {hybrid }}$ and goto Step 3.

Note that in step 3, for consistency, we use the same decision criteria outlined in Section 3.1.

\section{References}

Alfonsi, A., Cances, E., Turinici, G., Ventura, B. \& Huisinga, W. (2005), 'Adaptive simulation of hybrid stochastic and deterministic models for biochemical systems', ESAIM: Proceedings 14, 1-13.

Andrieu, C., Doucet, A. \& Holenstein, R. (2009), Particle Markov chain Monte Carlo for efficient numerical simulation, in P. L'Ecuyer \& A. B. Owen, eds, 'Monte Carlo and Quasi-Monte Carlo Methods 2008', Spinger-Verlag Berlin Heidelberg, pp. 45-60.

Andrieu, C., Doucet, A. \& Holenstein, R. (2010), 'Particle Markov chain Monte Carlo methods (with discussion)', Journal of the Royal Statistical Society Series B 72(3), 1269.

Andrieu, C. \& Roberts, G. O. (2009), 'The pseudo-marginal approach for efficient computation', Annals of Statistics 37, 697-725.

Beaumont, M. A. (2003), 'Estimation of population growth or decline in genetically monitored populations', Genetics 164, 1139-1160.

Beskos, A. \& Roberts, G. O. (2005), 'Exact simulation of diffusions', Annals of Applied Probability 15(4), 2422-2444.

Boys, R. J., Wilkinson, D. J. \& Kirkwood, T. B. L. (2008), 'Bayesian inference for a discretely observed stochastic-kinetic model', Statistics and Computing 18, 125-135.

Burrage, K., Tian, T. \& Burrage, P. (2004), 'A multi-scaled approach for simulating chemical reaction systems', Progress in Biophysics and Molecular Biology 85, 217-234.

Carpenter, J., Clifford, P. \& Fearnhead, P. (1999), 'An improved particle filter for nonlinear problems', IEE Procedings - Radar, Sonar and Navigation 146, 2-7.

Casella, B. \& Roberts, G. O. (2011), 'Exact simulation of jump-diffusion processes with Monte carlo applications', Methodology and Computing in Applied Probability 13(3), 449-473.

Del Moral, P. (2004), Feynman-Kac Formulae: Genealogical and Interacting Particle Systems with Applications, Springer, New York.

Del Moral, P., Jacod, J. \& Protter, P. (2002), 'The Monte Carlo method for filtering with discrete-time observations', Probability Theory and Related Fields 120, 346-368. 
Doucet, A., Godsill, S. \& Andrieu, C. (2000), 'On sequential Monte Carlo sampling methods for Bayesian filtering', Statistics and Computing 10, 197-208.

Doucet, A., Pitt, M. K. \& Kohn, R. (2013), Efficient implementation of Markov chain Monte Carlo when using an unbiased likelihood estimator. Available from http://arxiv.org/pdf/1210.1871.pdf.

Fearnhead, P., Sherlock, C. \& Giagos, V. (2014), 'Inference for biological networks using the linear noise approximation', To appear in Biometrics .

Gibson, M. A. \& Bruck, J. (2000), 'Efficient exact stochastic simulation of chemical systems with many species and many channels', Journal of Physical Chemistry A 104(9), 1876-1889.

Gillespie, D. T. (1977), 'Exact stochastic simulation of coupled chemical reactions', Journal of Physical Chemistry 81, 2340-2361.

Gillespie, D. T. (2000), 'The chemical Langevin equation', The Journal of Chemical Physics 113(1), 297-306.

Golightly, A. \& Gillespie, C. S. (2013), Simulation of stochastic kinetic models, in 'In Silico Systems Biology', Springer, pp. 169-187.

Golightly, A. \& Wilkinson, D. J. (2005), 'Bayesian inference for stochastic kinetic models using a diffusion approximation', Biometrics 61(3), 781-788.

Golightly, A. \& Wilkinson, D. J. (2011), 'Bayesian parameter inference for stochastic biochemical network models using particle Markov chain Monte Carlo', Interface Focus $\mathbf{1}(6), 807-820$.

Gordon, N. J., Salmond, D. J. \& Smith, A. F. M. (1993), 'Novel approach to nonlinear/nonGaussian Bayesian state estimation', IEE Proceedings-F 140, 107-113.

Guptasarma, P. (1995), 'Does replication-induced transcription regulate synthesis of the myriad low copy number proteins of Escherichia coli?', BioEssays 17, 987-997.

Haseltine, E. L. \& Rawlings, J. B. (2002), 'Approximate simulation of coupled fast and slow reactions for stochastic chemical kinetics', Journal of Chemical Physics 117(15), 69596969 .

Heron, E. A., Finkenstadt, B. \& Rand, D. A. (2007), 'Bayesian inference for dynamic transcriptional regulation; the Hes1 system as a case study', Bioinformatics 23, 25962603.

Higham, D., Intep, S., Mao, X. \& Szpruch, L. (2011), 'Hybrid simulation of autoregulation within transcription and translation', BIT Numerical Mathematics 51, 177-196.

Hobolth, A. \& Stone, E. A. (2009), 'Simulation from endpoint-conditioned, continuous-time Markov chains on a finite state space, with applications to molecular evolution', Annals of Applied Statistics 3(3), 1204. 
Kiehl, T. R., Matteyses, R. M. \& Simmons, M. K. (2004), 'Hybrid simulation of cellular behavior', Bioinformatics 20(3), 316-322.

Kitano, H. et al. (2001), Foundations of systems biology, MIT press Cambridge.

Komorowski, M., Finkenstadt, B., Harper, C. \& Rand, D. (2009), 'Bayesian inference of biochemical kinetic parameters using the linear noise approximation', BMC Bioinformatics 10(1), 343.

Lewis, P. A. W. \& Shedler, G. S. (1979), 'Simulation of a nonhomogeneous Poisson process by thinning', Naval Research Logistics Quaterly 26, 401-413.

McAdams, H. H. \& Arkin, A. (1999), 'Its a noisy business: Genetic regulation at the nanomolar scale', Trends in Genetics 15, 65-69.

Pahle, J. (2009), 'Biochemical simulations: stochastic, approximate stochastic and hybrid approaches', Briefings in Bioinformatics 10(1), 53-64.

Petzold, L. (1983), 'Automatic selection of methods for solving stiff and non-stiff systems of ordinary differential equations', SIAM J. Sci. Stat. Comp. 4(1), 136-148.

Picchini, U. (2013), 'Inference for SDE models via Approximate Bayesian Computation', Journal of Computational and Graphical Statistics . DOI:0.1080/10618600.2013.866048.

Pitt, M. K., dos Santos Silva, R., Giordani, P. \& Kohn, R. (2012), 'On some properties of Markov chain Monte Carlo simulation methods based on the particle filter', $J$. Econometrics 171(2), 134-151.

Pitt, M. K. \& Shephard, N. (1999), 'Filtering via simulation: Auxiliary particle filters', Journal of the American Statistical Association 446(94), 590-599.

Purutcuoglu, V. \& Wit, E. (2007), 'Bayesian inference of the kinetic parameters of a realistic MAPK/ERK pathway', BMC Systems Biol. 1.

R Core Team (2013), R: A Language and Environment for Statistical Computing, R Foundation for Statistical Computing, Vienna, Austria.

Rao, V. \& Teh, Y. W. (2013), 'Fast MCMC sampling for Markov jump processes and extensions', Journal of Machine Learning Research 14, 3207-3232.

Salis, H. \& Kaznessis, Y. (2005), 'Accurate hybrid stochastic simulation of a system of coupled chemical or biochemical reactions', Journal of Chemical Physics 122, 054103.

Sherlock, C., Thiery, A., Roberts, G. O. \& Rosenthal, J. S. (2013), On the effciency of pseudo-marginal random walk Metropolis algorithms. Available from http://arxiv.org/abs/1309.7209.

Stathopoulos, V. \& Girolami, M. (2013), 'Markov chain Monte Carlo inference for Markov jump processes via the linear noise approximation.', Phil. Trans. R. Soc. A. 371, 20110549. 
Swain, P. S., Elowitz, M. B. \& Siggia, E. D. (2002), 'Intrinsic and extrinsic contributions to stochasticity in gene expression', PNAS 99(20), 12795-12800.

van Kampen, N. G. (2001), Stochastic Processes in Physics and Chemistry, North-Holland.

Wickham, H. (2009), ggplot2: elegant graphics for data analysis, Springer New York.

Wilkinson, D. J. (2012), Stochastic Modelling for Systems Biology, 2 edn, Chapman and Hall/CRC Press, London. 